\title{
STUTTY: Aplicación móvil para mermar la tartamudez utilizando la técnica del "tartamudeo-sencillo"
}

\author{
Ricardo Solano Monje ${ }^{1}$, Nayeli Joaquinita Meléndez Acosta ${ }^{2}$, \\ Homero Vladimir Ríos Figueroa ${ }^{3}$ \\ ${ }^{1}$ Centro Veracruzano de Investigación y Posgrado, Xalapa, Veracruz, \\ México \\ ${ }^{2}$ Universidad del Istmo, Ciudad Ixtepec, Oaxaca, \\ México \\ ${ }^{3}$ Universidad Veracruzana, Xalapa, Veracruz, \\ México \\ rsolanomonje@gmail.com nayelimelendez@gmail.com hrios@uv.mx
}

\begin{abstract}
Resumen. Este artículo se enfoca en el desarrollo de una aplicación móvil (app) STUTTY, para ayudar en el problema del tartamudeo para niños de entre 6 y 11 años. Con el uso de STUTTY se pretende proporcionar una herramienta de apoyo al paciente en el proceso de tratamiento y prevención de la tartamudez. La aplicación móvil propuesta utiliza la técnica del "tartamudeo-sencillo", la cual está enfocada en separar la palabra en sílaba, con la diferencia susceptible de un alargamiento del sonido vocal que sigue a la consonante. Esta aplicación está dirigida al tipo de tartamudez clónica, en la cual existe una frecuencia en las repeticiones de sílabas, principalmente en consonantes, más en oclusivas y el momento de la tartamudez está presente al principio de la palabra. Para la realización de STUTTY se utilizó el modelo desarrollo incremental para identificar los servicios con prioridad y fueran los primeros en desarrollarse. Las pruebas muestran una efectividad del $73.6 \%$.
\end{abstract}

Palabras clave: tartamudez, aplicación móvil, tartamudeo-sencillo, consonantes oclusivas.

\section{STUTTY: Mobile Application for Diminishing Stuttering Using the "Simple Stuttering" Technique}

\begin{abstract}
This article focuses on the development of a Mobile App named STUTTY made to help with the stuttering problem for children between the ages of 6 and 11. STUTTY is intended to provide a tool to support the patient in the process of treatment and prevention of stuttering. The proposed mobile application uses the "simple-stuttering" technique, which is focused on separating words into syllables, susceptible to an extension of the vocal sound following the consonant. This application is directed to the type of cloned
\end{abstract}




\begin{abstract}
stuttering, in which there is a frequency in the repetitions of syllables, mainly in consonants, more in occlusive and the moment of stuttering is present at the beginning of the word. For the implementation of STUTTY, the incremental development model was used to identify the services with priority and were the first ones to be developed. The tests show an effectiveness of $73.6 \%$.
\end{abstract}

Keywords: stuttering, mobile application, simple stuttering, consistent occlusion.

\title{
1. Introducción
}

La tartamudez es una dificultad del habla que muchas veces se presenta cuando se habla en público. Hay más hombres que mujeres con este problema, en una proporción de 4 a 1 y comúnmente inicia en la infancia entre 2 a 5 años. La tartamudez no es una enfermedad, es involuntaria y aparece y desaparece por periodos de tiempo variables [1].

En la infancia los niños empiezan a tartamudear, aunque la mayoría de las veces este problema desaparece sólo, sin la ayuda de un profesional, si la dificultad continúa después de un año desde su comienzo, existe el riesgo de que el problema se mantenga [2]. Por ello es conveniente realizar una intervención temprana que ayude a prevenir el problema con una terapia adecuada, que ayude a los niños a conseguir un habla fluida, para que la dificultad no persista.

Todas las personas tartamudean de diferente forma, algunas repiten sílabas o palabras, otras alargan sonidos y otras cuando hablan o antes de terminar una palabra se bloquean [3], [1].

Esta aplicación tiene como objetivo fundamental proporcionar una herramienta de apoyo al paciente en el proceso de tratamiento y prevención de la tartamudez.

Este artículo presenta el desarrollo de una aplicación para dispositivos móviles, que ayuda en el tratamiento de la tartamudez clónica en niños entre 6 y 11 años, utilizando la técnica "tartamudeo-sencillo" de Ann en [4]. El contenido del artículo se encuentra organizado de la siguiente manera, en la segunda sección se presenta el marco teórico respecto a la tartamudez y se describen algunas aplicaciones que se han implementado para tratar dicho problema, luego en la tercera sección se explica el funcionamiento de la técnica "tartamudeo-sencillo" utilizada para el desarrollo de la aplicación móvil propuesta, posteriormente en la cuarta y quinta sección se presenta la metodología utilizada y la implementación de la aplicación móvil STUTTY, enseguida se muestran las pruebas realizadas y finalmente en la sexta sección se presentan las conclusiones y trabajo futuro.

\section{La tartamudez}

Tartamudeo es la interrupción en la fluidez normal del habla, que afecta la comunicación y se acompaña de tensión muscular, miedo y estrés [3].

En la tartamudez intervienen muchos factores tales como los neurológicos, psicológicos y sociales, la tartamudez afecta entre $1,5 \%$ y $2 \%$ de la población adulta 
[3]. Esta alteración en la fluidez del habla tiene un gran impacto en la calidad de vida y en el rendimiento escolar, laboral y social [1], [5]. Los niños de preescolar que tartamudean son menos precisos en tareas implícitas de respuesta verbal y exhiben tiempos de reacción más lentos que los niños que no tartamudean [6]. Esto no significa que sean menos inteligentes, pero si afecta su rendimiento escolar.

El tartamudeo se presenta de manera diferente en cada persona. Alguna persona tendrá problemas en un conjunto de palabras mientras que otra los tendrá en otro diferente. Otras personas tartamudean cuando hablan en voz alta, pero no lo hacen cuando nadie los escucha, algunas otras tartamudean cuando nadie los escucha y hablan fluido cuando hablan en voz alta [4].

Otro aspecto importante es que las personas que tartamudean por lo regular hablan muy rápido, quizás porque quieren terminar pronto la lectura, antes de que les afecte el tartamudeo. Pero hablar rápido estorba más que ayudar.

\subsection{Tartamudez en niños}

La tartamudez inicia con frecuencia alrededor de los 2 años, sin que exista un motivo desencadenante [7], pero con frecuencia desaparece sin la ayuda de un especialista. La tartamudez puede identificarse a tiempo para prevenir consecuencias de su persistencia.

Aproximadamente el $80 \%$ de los tartamudos son niños [8]. Además la mayoría de los tartamudos comenzaron a tartamudear en la niñez, es decir, el $90 \%$ de casos de tartamudeo iniciaron antes de los 4 años.

Cuando un niño tartamudea es necesario no ponerle atención a su tartamudeo, si se le demuestra ansiedad o se le hace sentir que su lenguaje es inadecuado, se le induce a evitar el tartamudeo [4]. El niño debe saber que es libre de tartamudear, no hay que presionarles a hablar con fluidez.

La tartamudez temprana se presenta principalmente en repeticiones de sílabas y palabras. En la tartamudez existe algo llamado momento del tartamudeo, que es el instante en el que ocurre el tartamudeo durante el habla, algunos de estos pueden ser: repetición de palabra, repetición de sonidos o sílabas, repetición de frases, frases incompletas, palabras rotas y prolongación [5].

\subsection{Momentos de la tartamudez}

Por lo general los momentos de tartamudez en el lenguaje no suceden al azar, con frecuencia se presenta al principio de una palabra o al iniciar una frase [4], [5].

Los momentos se pueden clasificar en tres principalmente [5]:

La consonante inicial de la palabra. La presencia de tartamudez es más frecuente en consonantes y en un $90 \%$ en sonidos iniciales de las palabras

La primera palabra de una frase. Así como ocurren en la primera sílaba de una palabra, también ocurre en la primera palabra de una frase.

Al final de las palabras. Es muy raro, pero en ocasiones también ocurre el tartamudeo al final de las palabras [9]. 


\subsection{División de los sonidos}

Los sonidos se dividen en dos vocales y consonantes, estos últimos pueden dividirse a su vez en dos grupos oclusivas y continuas [4].

En las consonantes oclusivas debemos hacer explotar algo antes de oír el sonido, estas son seis: "p", "b", "t", "d", "k" y "g".

Las consonantes continuas son aquellas que se pueden decir hasta que se acabe el aliento y son el resto de contantes.

\subsection{El tartamudeo en sonidos determinados}

Por lo regular el tartamudeo no ocurre en un solo grupo de sonidos, sino en varios. La mayoría de las veces el problema del tartamudeo se encuentra en las consonantes oclusivas [4]. Los sonidos para el análisis del tartamudeo se pueden agrupar en cinco: vocales, oclusiva, nasales, semivocales y continuas restantes, ver Fig.1.

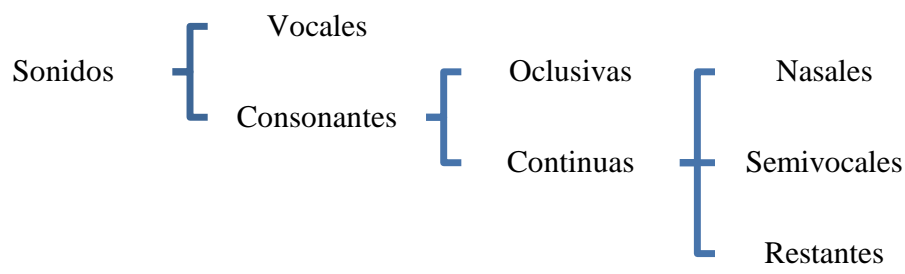

Fig. 1. División de los sonidos en el análisis del tartamudeo [4].

Los sonidos nasales son " $m$ ", " $n$ " y "ñ", estos sonidos provienen de la nariz en lugar de la boca.

Los sonidos semivocales son dos "y" y "w", estos sonidos inician con una vocal y se deslizan a otro sonido vocal.

\subsection{Clasificación de la tartamudez}

La tartamudez se puede clasificar según su origen o su manifestación [1]:

Según su origen puede ser neurológica causada por una lesión del cerebro, psicógena producida por un trauma difícil y de desarrollo que ocurre cuando el niño comienza hablar.

Según su manifestación puede ser tónica que se presenta cuando hay interrupciones por espasmos, clónica cuando existe una frecuencia en las repeticiones de sílabas principalmente en consonantes, más en oclusivas y está presente al principio de la palabra y tónico-clónica o mixta esta es una combinación de las dos anteriores, además la fluidez del habla es lenta y de repente se acelera. 


\subsection{Técnicas de la tartamudez}

Please note that the first paragraph of a section or subsection is not indented. The first paragraphs that follows a table, figure, equation etc. does not have an indent, either. Existen numerosas técnicas para el control de tartamudez, algunas de ellas son [4]:

Seguimiento. Esta técnica funciona de la siguiente forma, se tiene grabada una cinta con un monólogo de otra persona, el paciente con tartamudeo la escucha utilizando audífonos y trata de producir lo que oye con voz suave, de esta forma habla y escucha al mismo tiempo. En algunas personas ha tenido éxito esta técnica.

Retroalimentación Auditiva Demorada (DAF, Delayed Auditory Feedback). El objetivo es que el paciente escuche sus propias palabras a través de audífonos, con un retraso de más o menos un quinto de segundo. Su eficiencia varía, pero en algunos casos el tartamudeo disminuye.

Habla Silábica. El paciente debe usar el mismo tiempo y dar el mismo énfasis a cada sílaba. Es muy fácil de aprender. Tiene mejores resultados cuando se aplica con intensidad.

Metrónomo electrónico. En esta técnica el paciente lleva unos audífonos que le transmiten una señal a sus oídos, el objetivo es hacer coincidir el énfasis de su habla con la señal. El paciente no requiere la asistencia constante de un terapeuta como en las otras terapias, el paciente sólo requiere el metrónomo y las instrucciones y puede continuar la terapia.

Encubrimiento (masker). En esta técnica la voz del paciente es disfrazada, lo que significa que el paciente es incapaz de escuchar sus propias palabras. Ya existe un aparato electrónico llamado Masker, al utilizarlo no es necesario realizar un esfuerzo para mejorar el tartamudeo. En algunas personas sirve en otras no.

Terapia de Johnson. Cuando existe temor al tartamudeo se trata de evitar las palabras, los sonidos y situaciones que se temen. El paciente pretende evitar el tartamudeo, pero para controlarlo es necesario dejar de evitarlo. Está terapia no va dirigida a romper los síntomas del tartamudeo, sino todo lo contrario a ganar el control del mismo, es decir aprender exactamente como se tartamudea y luego se debe tartamudear a propósito.

Algunas de las técnicas más usadas en aplicaciones móviles son retroalimentación auditiva demorada, donde el usuario escucha su voz con un poco de retraso, el metrónomo en esta técnica el usuario debe pronunciar cada sílaba en determinado tiempo y la técnica del espejo, en la cual el usuario observa su conducta mientras practica sílabas.

\subsection{Trabajos relacionados}

Algunas aplicaciones que se han implementado para tratar el problema de la tartamudez son:

Ernest Stuttering App. Esta aplicación está diseñada para niños de edad escolar y adolescentes que tartamudean. Implementa cuatro juegos llamados "Hablando", "Identificando", "Memorizando" y "Dibujando". Además permite a los terapeutas trabajar con otros trastornos del habla. Pero no es gratuita [10]. 
Fluxxy. Es una aplicación móvil controlada por medio de la voz, diseñada para niños mayores de 6 años con tartamudez. Ayuda a entrenar el manejo de la voz y la respiración lúdica. Es gratuita, pero por el momento solo se encuentra en alemán [11].

Cure The Stuttering Free. Esta aplicación implementa la técnica de retroalimentación con retraso auditivo, graba la voz del paciente y su voz se reproduce con un retardo. Esta es la versión libre que limita a 60 segundos de uso continuo [12].

Sttuter Help. Esta aplicación móvil permite controlar la fluidez del habla y puede ser utilizado como parte de una terapia del habla, algunas de las técnicas que implementa son el metrónomo, el espejo y la retroalimentación con retraso auditivo. No es gratuita [13].

Stutter Rater. Es un contador de tartamudeo simple, esta aplicación es usada para diagnosticar y muestra el porcentaje de tartamudez por sílaba, así como sílabas por minuto. Es gratuita [14].

En su mayoría las aplicaciones móviles para ayudar a la tartamudez son de uso comercial y ninguna ha implementado la técnica del "tartamudeo-sencillo".

\section{Técnica "tartamudeo-sencillo"}

La técnica de tartamudeo utilizada en el desarrollo de la aplicación es "tartamudeosencillo", que consiste en pronunciar una palabra de forma natural, con la diferencia susceptible de colocar un alargamiento del sonido vocal que sigue a la consonante [4].

El funcionamiento de la técnica consiste en realizar una pronunciación adecuada de las palabras: la consonante se debe pronunciar suave, luego se debe deslizar sobre el sonido vocal el cual se debe prolongar (no separar ambos sonidos), y terminar con un sonido normal si es que existen más sílabas. En la Tabla 1 se muestra la técnica utilizando seis palabras con iniciales oclusivas:

Tabla 1. Tartamudeo-sencillo en consonantes oclusivas.

\begin{tabular}{llll}
\hline Palabra & Contante oclusiva & Sonido Vocal & Restante \\
\hline Piedra & (Suave) $\ll \mathrm{p} \gg$ & Prolongado $\ll$ ie $\gg$ & Normal $\ll$ dra $\gg$ \\
Bolsa & $($ Suave $) \ll \mathrm{b} \gg$ & Prolongado $\ll$ ol $\gg$ & Normal $\ll$ sa $\gg$ \\
Taco & $($ Suave $) \ll \mathrm{t} \gg$ & Prolongado $\ll \mathrm{a} \gg$ & Normal $\ll$ co $\gg$ \\
Dos & $($ Suave $) \ll \mathrm{d} \gg$ & Prolongado $\ll$ os $\gg$ & \\
Coco & $($ Suave $) \ll \mathrm{c} \gg$ & Prolongado $\ll 0 ~$ & Normal $\ll$ co $\gg$ \\
Ganso & $($ Suave $) \ll \mathrm{g} \gg$ & Prolongado $\ll$ an $\gg$ & Normal $\ll$ so $\gg$ \\
\hline
\end{tabular}

Ahora vemos las oclusivas seguidas por otra consonante, en estas palabras aparecen dos consonantes seguidas antes del sonido vocal, en este caso no se alarga la segunda consonante porque se distorsiona el sonido de la palabra, en estos casos se consideran las dos primeras consonantes como una sola unidad con un sonido suave, ver Tabla 2. 
STUTTY: Aplicación móvil para mermar la tartamudez utilizando la técnica del ...

Tabla 2. Tartamudeo-sencillo en consonantes oclusivas seguidas por otra consonante.

\begin{tabular}{llll}
\hline \multicolumn{1}{c}{ Palabra } & Contante oclusiva & Sonido Vocal & \multicolumn{1}{c}{ Restante } \\
\hline Premio & $($ Suave $) \ll \mathrm{pr} \gg$ & Prolongado $\ll \mathrm{e} \gg$ & Normal $\ll$ mio $\gg$ \\
Blusa & $($ Suave $) \ll \mathrm{bl} \gg$ & Prolongado $\ll \mathrm{u} \gg$ & Normal $\ll \mathrm{sa} \gg$ \\
Tractor & $($ Suave $) \ll \mathrm{tr} \gg$ & Prolongado $\ll \mathrm{ac} \gg$ & Normal $\ll$ tor $\gg$ \\
Dragón & $($ Suave $) \ll \mathrm{dr} \gg$ & Prolongado $\ll \mathrm{a} \gg$ & Normal $\ll$ gón $\gg$ \\
Clavo & $($ Suave $) \ll \mathrm{cl} \gg$ & Prolongado $\ll \mathrm{a} \gg$ & Normal $\ll$ vo $\gg$ \\
Globo & $($ Suave $) \ll \mathrm{gl} \gg$ & Prolongado $\ll \mathrm{o} \gg$ & Normal $\ll$ vo $\gg$ \\
\hline
\end{tabular}

La técnica de "tartamudeo-sencillo" se ha seleccionada debido a su simplicidad y porque ha mostrado en la práctica empírica buenos resultados en los pacientes que realmente trabajan para asimilarlo. La aplicación implementada en este trabajo está enfocada en la tartamudez clónica, que es un tipo de tartamudez muy común. Además de que esta técnica no ha sido implementada en ninguna aplicación móvil.

\section{Metodología}

\subsection{Desarrollo incremental}

La metodología utilizada para la implementación de esta aplicación móvil es desarrollo incremental. En esta metodología los clientes identifican de manera somera los requerimientos de la aplicación y se les establecen prioridades, de esta forma se entregan primero los de mayor prioridad, además eso significa que el usuario encontrará menos fallas en las tareas más importantes de la aplicación [15].

Una vez definidos los requerimientos se definen los incrementos. En cada incremento esta metodología permite entregar un producto operacional, aunque muchas de las veces los primeros incrementos son versiones incompletas.

Esta metodología ha sido seleccionada porque permite que el usuario experimente con la aplicación ayudando a clarificar sus requerimientos para los incrementos posteriores. Además de que el usuario puede seguir agregando más especificaciones.

\subsection{Análisis de requerimientos}

Esta subsección se centra en la descripción de los requerimientos funcionales, la identificación de su prioridad y la definición de los incrementos, pasos necesarios en el modelo de desarrollo incremental. La definición de los requerimientos iniciales se realiza utilizando dos técnicas, mediante entrevistas con el cliente, especialista en trastornos del habla y mediante estudios de documentación respecto al funcionamiento de la técnica "tartamudeo sencillo".

Existen diversos aspectos a tomar en consideración para priorizar los requerimientos, la técnica utilizada para priorizar los requerimientos de la aplicación es ranking, donde 
se asigna una posición a cada requerimiento que va de 1 a $\mathrm{N}$, siendo $\mathrm{N}$ la cantidad de requerimientos y 1 el requerimiento de mayor importancia.

Tabla 3. Requerimientos iniciales de la aplicación.

\begin{tabular}{|c|c|c|c|}
\hline No. & Requerimientos & Prioridad & Incremento \\
\hline 1 & $\begin{array}{l}\text { La aplicación debe permitir al usuario elegir un tipo de } \\
\text { consonante oclusiva, nasal, semivocal, continua o vocal } \\
\text { a practicar. }\end{array}$ & 6 & 3 \\
\hline 2 & $\begin{array}{l}\text { Al seleccionar una consonante o vocal, la aplicación } \\
\text { debe mostrar una lista de palabras a practicar. }\end{array}$ & 7 & 3 \\
\hline 3 & $\begin{array}{l}\text { La lista debe contener } 10 \text { palabras a practicar, } \\
\text { seleccionadas de forma aleatoriamente. }\end{array}$ & 5 & 2 \\
\hline 4 & $\begin{array}{l}\text { Al seleccionar una palabra se debe escuchar la } \\
\text { pronunciación usando la técnica de "tartamudeo- } \\
\text { simple". }\end{array}$ & 1 & 1 \\
\hline 5 & $\begin{array}{l}\text { La aplicación debe permitir volver a escuchar de nuevo } \\
\text { la palabra. }\end{array}$ & 2 & 1 \\
\hline 6 & La aplicación debe permitir pasar a la siguiente palabra & 3 & 1 \\
\hline 7 & Debe permitir regresar a la lista de palabras & 4 & 2 \\
\hline
\end{tabular}

Los requerimientos iniciales, su prioridad y los incrementos utilizados en la elaboración de la aplicación están definidos en la Tabla 3.

En la Tabla 3 se puede observar que se definen siete requerimientos iniciales y 3 incrementos, los cuales se conforman por dos o tres requisitos. Podemos ver que el primer incremento cumple con los requerimientos más importantes.

\subsection{Diseño}

En esta fase se describe brevemente el diseño. En la Fig. 2, se muestra el caso de uso general de la aplicación, donde se pueden apreciar las acciones más importantes que el usuario podrá realizar al usar la aplicación:

\subsection{Desarrollo}

Una "Actividad" es el nombre dado a una sola ventana de la aplicación móvil con la que el usuario puede interactuar en un momento dado, a partir de aquí hablaremos de actividad refiriéndonos a una ventana. En esta sección se mostrarán las interfaces realizadas en el entorno de desarrollo Android Studio. La aplicación implementada fue probada en un teléfono inteligente Android versión Lollipop 5.1. La fig. 3 (a) muestra la ventana principal de inicio de la aplicación STUTTY, aquí el usuario debe seleccionar el botón de "INICIO".

STUTTY cuenta con 5 módulos: 4 de tipo consonante y uno para las vocales (Ver Fig. 3 (b)). Las consonantes oclusivas se colocaron en primer lugar debido a que la 
aplicación está enfocada a la tartamudez clónica, donde el problema frecuentemente se presenta en este tipo de consonantes.

La Fig. 4 (a) muestra la lista de palabras a practicar de la letra seleccionada, esta lista es dinámica, cada vez que el usuario entra las palabras cambian de forma aleatoria.

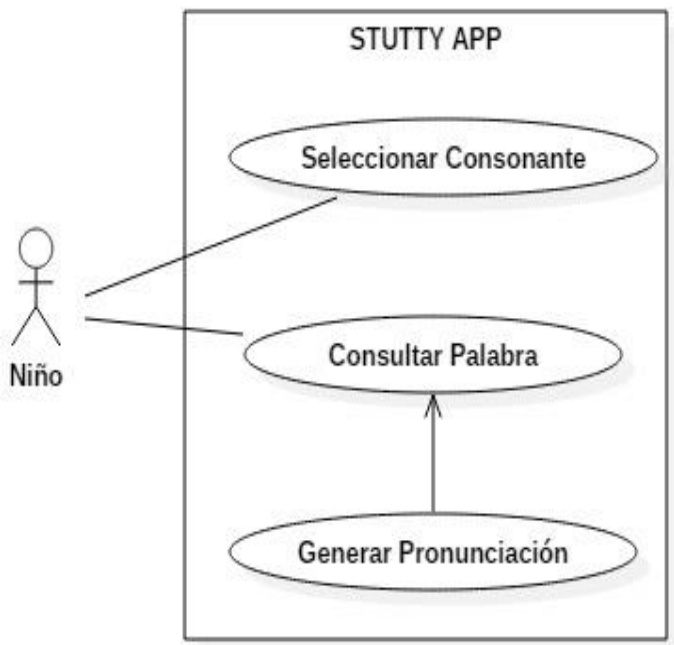

Fig. 2. Diagrama de Casos de uso de la aplicación.
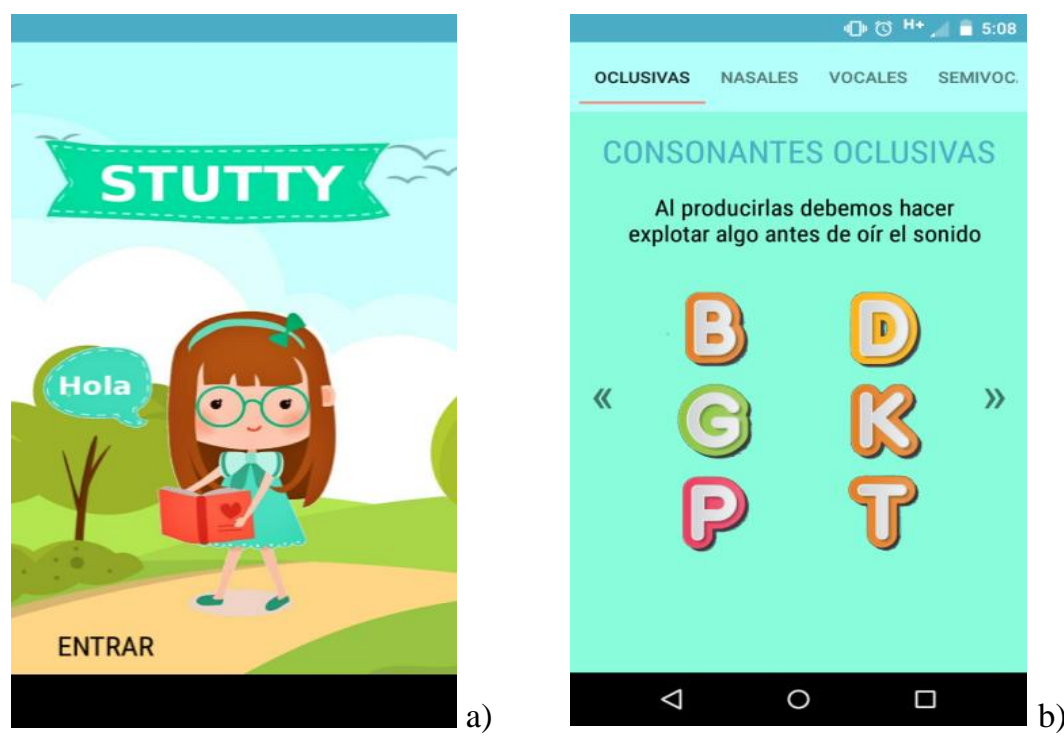

Fig. 3. a) Actividad de inicio de la aplicación STUTTY. b) Actividad para seleccionar el tipo de consonante. 

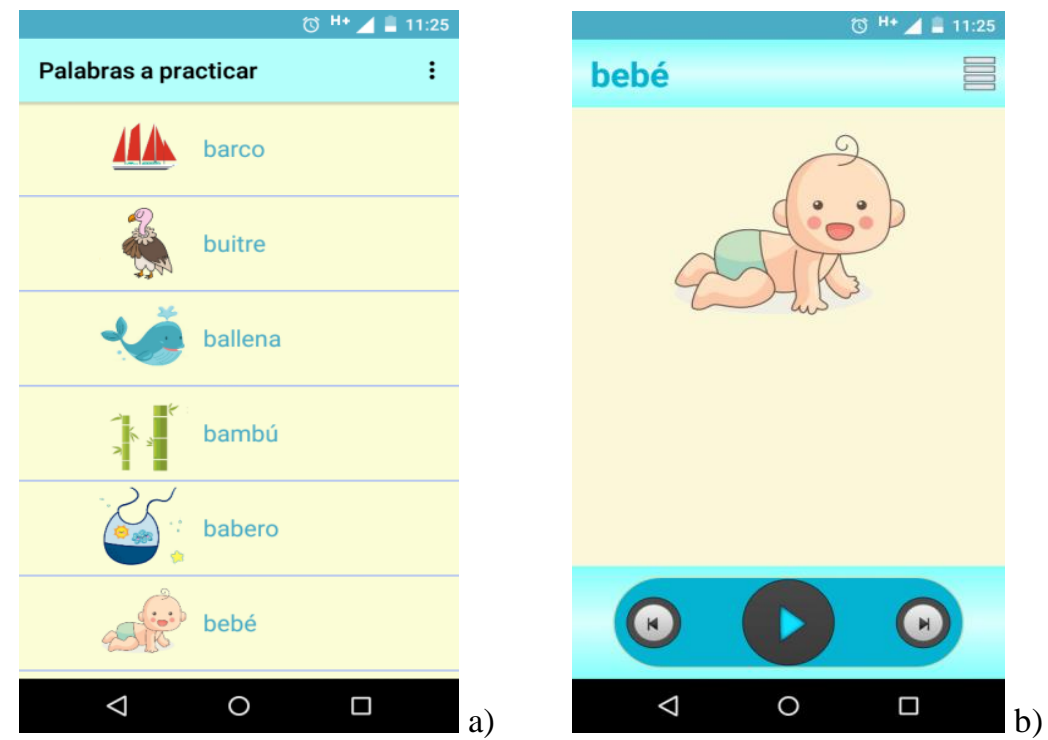

Fig. 4. a) Actividad que muestra la lista de palabras a practicar. b) Actividad para escuchar la pronunciación usando la técnica "tartamudeo-sencillo".

La aplicación STUTTY permite al usuario tantas veces necesite y quiera escuchar la pronunciación de la palabra, usando la técnica del "tartamudeo-sencillo" (Ver Fig. 4(b)). También podrá ir a la siguiente palabra o regresar a la palabra anterior, además de poder retornar a la lista de palabras para seleccionar otra en particular.

\subsection{Pruebas}

En las pruebas realizadas para evaluar la fluidez del habla en los usuarios, se mide tomando en cuenta dos variables: frecuencia y cantidad. La variable frecuencia mide cuantas palabras en un texto pronuncia con alteraciones y cantidad mide cuantas veces una palabra, sílaba o sonidos son repetidos.

En la prueba participaron 4 usuarios (niños entre 6 y 11 años), de los cuales uno tiene tartamudez establecida inicial y tres con tartamudez normal. La prueba consistió en leer 10 frases antes de usar la aplicación, un mes después de utilizar la aplicación el usuario volvió a leer otras 10 frases. Los resultados se muestran en la siguiente sección.

\section{Resultados}

Las pruebas realizadas se describieron en la sección anterior, a continuación se muestran los resultados obtenidos. La Tabla 4 presenta promedios de frecuencia y cantidad al evaluar a cuatro usuarios. 
STUTTY: Aplicación móvil para mermar la tartamudez utilizando la técnica del ...

En la Tabla 4 se observa que hubo una pequeña mejoría, está claro que es necesario tiempo y uso contante de la aplicación para tener mejores resultados, como los obtenidos en la práctica empírica al utilizar la técnica "tartamudeo-sencillo" [4].

Tabla 4. Promedios de frecuencia y cantidad sobre 10 frases.

\begin{tabular}{ccccc}
\hline \multirow{2}{*}{ Usuario } & \multicolumn{2}{c}{ Antes } & \multicolumn{2}{c}{ Después } \\
\cline { 2 - 5 } & Frecuencia & Cantidad & Frecuencia & Cantidad \\
\hline 1 & 0.13 & 0.10 & 0.10 & 0.09 \\
2 & 0.12 & 0.11 & 0.09 & 0.09 \\
3 & 0.12 & 0.10 & 0.10 & 0.10 \\
4 & 0.20 & 0.13 & 0.18 & 0.12 \\
\hline
\end{tabular}

En las pruebas se ha comprobado que la aplicación ayuda al usuario a realizar su práctica diaria con mayor interés, incluso recuerda que debe practicar.

El resultado de efectividad total del software fue de $73.6 \%$, el cual es un promedio de la efectividad de cada usuario. Durante las pruebas realizadas la utilización de aplicación STUTTY en niños de entre 6 y 11 tiene una aceptación muy favorable, los niños se interesan en seguir practicando, les agrada escuchar la voz y repiten continuamente las palabras como se les indica.

La intervención temprana se realiza al fomentar el uso de la de la aplicación STUTTY, asumiendo que en algunos casos no hace falta.

\section{Conclusiones y trabajo futuro}

En este trabajo se ha desarrollado una aplicación móvil llamada STUTTY, para ayudar en el tratamiento de tartamudez clónica para niños entre 6 y 11 años, utilizando la técnica "tartamudeo-sencillo".

La aplicación STUTTY incentiva a que los niños practiquen la técnica "tartamudeo -sencillo", además esta aplicación puede ser usada en niños aunque no tengan tartamudez, lo cual nos permite realizar una intervención temprana para prevenir futuros problemas.

La aplicación tiene una aceptación muy favorable, los niños se interesan en seguir practicando, les agrada escuchar la pronunciación y repiten la palabra como se les indica.

Como trabajo futuro se pretende agregar tipos de usuario, ya que en estos momentos la aplicación aun no contiene un registro de utilización. También se pretende agregar más actividades para practicar el "tartamudeo-sencillo".

Por otro lado otros componentes de la fluidez que serán tomados en cuenta en próximas pruebas serán: el ritmo, la velocidad, la prosodia, la secuencia y la fluencia. 


\section{Referencias}

1. Gutiérrez-Ornia, J.: Aceptando la tartamudez. Universidad de Valladolid (2015)

2. Yairi, E.: Disfluency characteristics of childhood stuttering. Nature and Treatment of Stuttering: New Directions, Needham Height, Allyn \& Bacon (1997)

3. Groesman, C. P.: Tartamudez, guía para docentes. España: Fundación española de la tartamudez

4. Ann, I.: Cómo vencer el tartamudeo. Mondadori, Grijalbo (2001)

5. Onslow, M.: Stuttering and its Treatment. Eleven Lectures, Sydney, Australian Stuttering Research Centre The University of Sydney (2017)

6. Anderson, J. D., Wagovich, S. A.: Explicit and Implicit Verbal Response Inhibition in Preschool-Age Children Who Stutter. Journal of Speech, Language, and Hearing Research, Vol. 60, pp. 836-852 (2017)

7. Sangorrín, J.: Disfemia o tartamudez. Revista de Neurología (2005)

8. Kehoe, T. D.: No Miracle Cures, A Multifactoral Guide to Stuttering Therapy (2008)

9. MacMillan, V., Kokolakis, A., Sheedy, S., Packman, A.: End-word dysfluencies in your children: A clinical report. Folia Phoniatrica et Logopaedica, pp. 115-125 (2014)

10. Gaiolas, M., Cintra, Z., de Ler, C.: Ernest, Stuttering App. 12 julio 2017, [En línea], Disponible en: https://play.google.com/store/apps/details?id=toymobi.ernesto\&hl=en

11. Institut der Kasseler Stottertherapie: FLUXXY. 12 julio 2017, [En línea], Disponible en: http://www.kasseler-stottertherapie.de/stottertherapie/smartphone-app-fluxxy/

12. Cure The Stuttering Free. 12 julio 2017, [En línea], Disponible en: https://play.google.com/ store/apps/details?id=jp.ne.nama.android.curethestutteringfree\&hl=en

13. Stutter Help. 12 julio 2017, [En línea], Available: https://play.google.com/store/apps/details? id=com.pcp.stutterhelp\&hl=en

14. Stutter Rater. 12 julio 2017, [En línea], Disponible en: https://play.google.com/store/apps/ details?id=com.oatau. stutter\&hl=en

15. Pressman, R. S.: Ingeniería de Software. Un enfoque práctico, México, McGRAW-HILL (2010) 